A Platinum Open Access Journal for Organic Chemistry

Paper

Free to Authors and Readers

DOAJ Seal

Arkivoc 2021, part viii, 204-216

\title{
Copper-catalyzed radical oxyallylation of olefins for the construction of alkene-containing isoxazolines
}

\author{
Xiaoxue He, Lijie Qian, Yuyu Dai, Xinhuan Yan, Xiaoqing Li,* and Xiangsheng Xu* \\ College of chemical Engineering, Zhejiang University of Technology, Hangzhou 310014, P. R. China \\ Email: xqli@zjut.edu.cn, future@zjut.edu.cn
}

Received 02-12-2021

Accepted Manuscript 04-26-2021

Published on line $06-06-2021$

\section{Abstract}

A radical-mediated approach to alkene oxyallylation using allylic oximes is described. The reaction proceeds under copper-catalytic redox-neutral conditions and tolerates various functional groups. This protocol thus enables the synthesis of structurally valuable isoxazolines and the introduction of a versatile olefin motif in a single step.<smiles>[R]C=C([R])C([R])/C([R])=N\O</smiles><smiles>[R]C1=NOC([R])(C([R])CC(=C)C#N)C1[R]</smiles>

Keywords: allylic oximes, copper-catalytic, oxyallylation, isoxazolines, allyl sulfones 


\section{Introduction}

The direct difunctionalization of alkenes, a valuable and readily available feedstock, represents one of the most effective strategies for increasing molecular complexity in synthetic organic chemistry. ${ }^{1-16}$ Among them, the oxyallylation of alkenes is a particularly useful reactions, because the alkenes motif in the resulting products offer rich possibilities for synthetic manipulations. Despite significant advances in the oxyfunctionalization of alkenes, ${ }^{17-26}$ oxyallylation of alkenes have been little explored. France and co-workers developed the palladium-catalyzed oxyallylation of unactivated alkenes using phenols, alcohol and carboxylic acid as the nucleophiles (Scheme 1a). ${ }^{27}$ However, monosubstituted alkene are not tolerated under the given reaction conditions, because of the 6 -hydride elimination of the putative $\sigma$-alkyl $\operatorname{Pd}(I I)$ intermediate. Alternatively, Alexanian and co-workers demonstrated the radical-mediated oxyallylation of alkenes with allyl sulfones using hydroxamic acids as the radical precursor with the assistance of $\mathrm{PhSO}_{2} \mathrm{NH}_{2}$ (Scheme 1b). ${ }^{28}$ Although highly practical, the types of oxygen-centered radicals remain limited. Encouraged by the works of Han, ${ }^{29,30}$ Wang, $^{31}$ and our group ${ }^{32}$ on the copper-catalyzed iminoxyl radical-mediated oxyfunctionalization of alkenes, we envisioned that iminoxyl radical may also be involved in the oxyallylation with allyl sulfones. Herein, we describe more convenient method, which has led to the construction of useful isoxazolines ${ }^{33-43}$ and the installation of versatile alkenyl groups in one transformation. It is expected that the resulting $\mathrm{C}=\mathrm{C}$ bond will serve as a useful precursor for obtaining other isoxazoline-containing compounds.

Palladium-catalyzed oxyallylation of alkenes

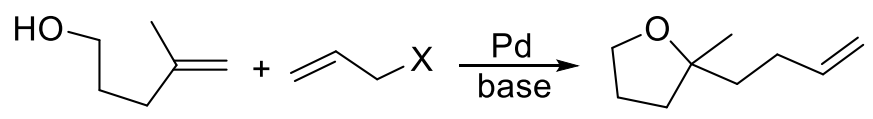

Radical-mediated oxyallylation of alkenes

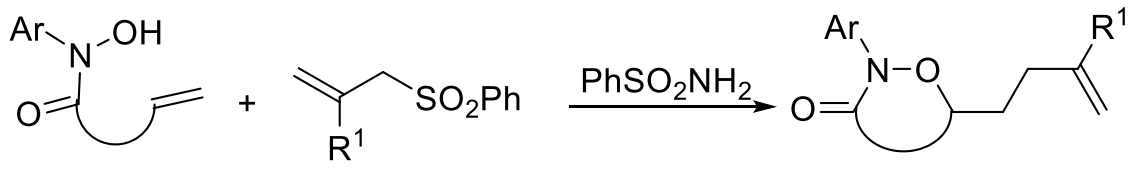

This work

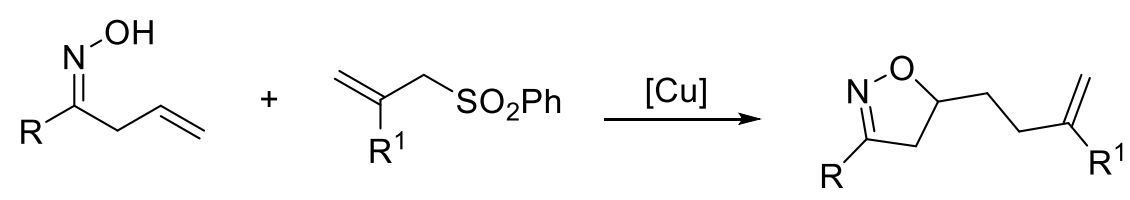

Scheme 1. Oxyallylation of alkenes.

\section{Results and Discussion}

The initial experiments were carried out with allylic oxime $\mathbf{1 a}$ and allyl sulfone $\mathbf{2} \mathbf{a}$ as the model substrates (Table 1 and S1). When the standard reaction conditions developed by our previous work revealed that using $\left(\mathrm{Cu}(\mathrm{OTf})_{2}, 1,10\right.$-phen and $\mathrm{Na}_{2} \mathrm{CO}_{3}$ were used as catalyst, ligand and base respectively in $\mathrm{MeCN}$ at $80{ }^{\circ} \mathrm{C}$ ), the desired isooxazoline 3 a was produced in $57 \%$ NMR yield. Inspired by these result, various reaction parameters were changed to explore the sensitivity of this reaction. $\mathrm{Cu}(\mathrm{II})$ catalysts were generally more effective than $\mathrm{Cu}(\mathrm{l})$ sources (entries 1-3 vs entries 4-6). Bpy was also effective ligand (entry 7), but the reaction yield diminished with TMEDA (entry 8). Other solvents, such as DCE, EtOH, toluene, THF and DMF uniformly less 
effective than MeCN (entries 9-13). Importantly, the loading of 2 a had significant effect on the reactions. Increasing the loading to 3 equiv provides $3 a$ in $84 \%$ NMR yield (entry 14 ). It is noteworthy that the remaining $2 \mathrm{a}$ could be recovered quantitatively. Finally, the reaction temperature could be reduced to $60{ }^{\circ} \mathrm{C}$ without the loss the reaction efficiency (entry 15). In the absence of $\mathrm{Cu}(\mathrm{OTf})_{2}$ and base, the reactions could hardly occur (entries 16-17), while control experiment lacking ligand was less effective (entry 18).

Table 1. Optimization of reaction conditions ${ }^{a, b}$

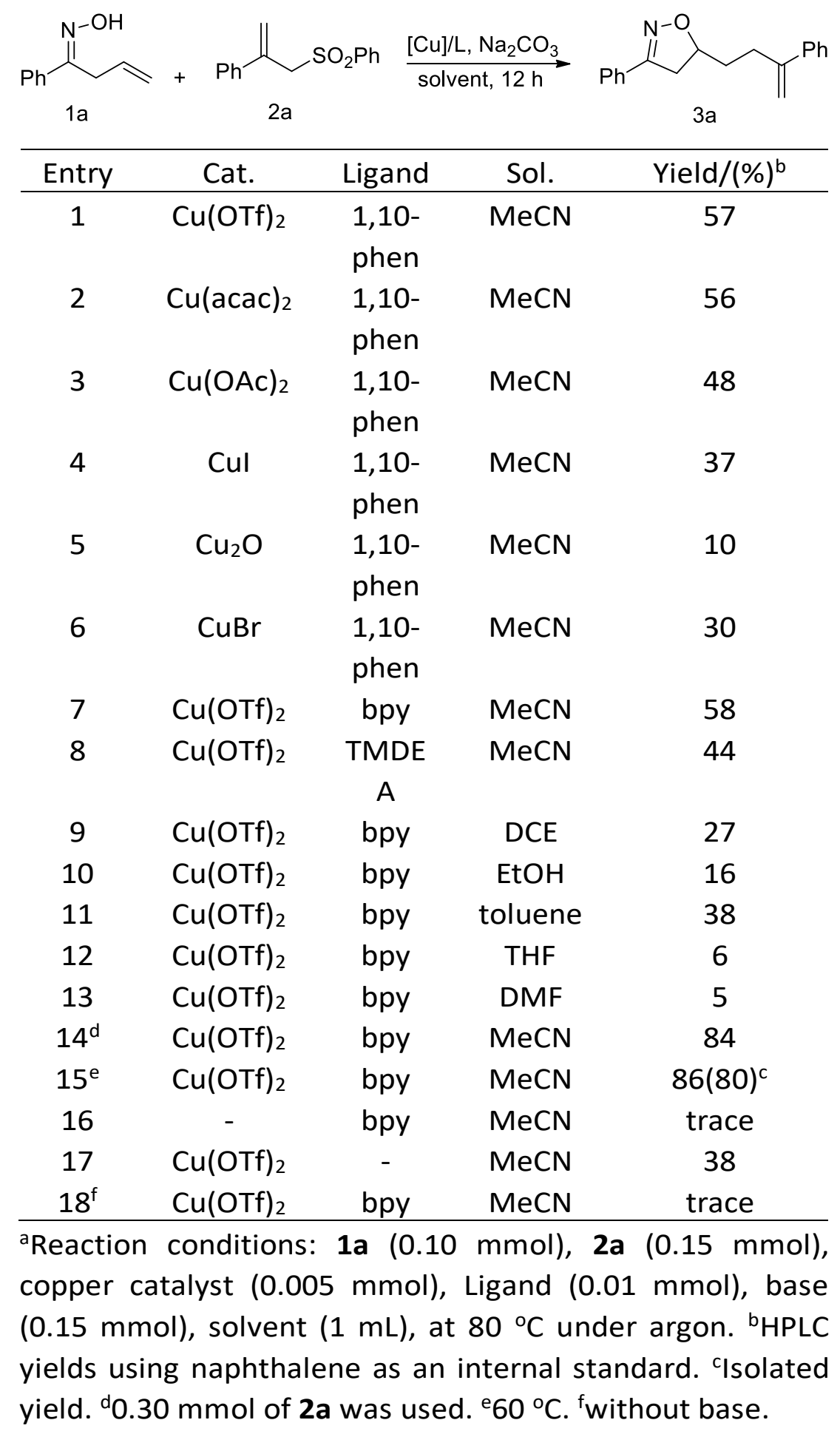


With the optimized conditions in hand, we next tested the reaction scope with respect to the allylic oximes, keeping allyl sulfone $\mathbf{2 a}$ as the substrate (Scheme 2). Allylic oximes bearing various electronically different substituents (methyl, methoxyl, fluoro, chloro, bromo, trifluorormethyl, nitro, and ester groups) proved to be suitable substrates and provided products $\mathbf{3 b - j}$ in moderate yields. In addition, naphthyl and thien-2-yl oxime were converted smoothly to the corresponding products (3k-I). Importantly, aliphatic oxime, such as phenethyl oxime was suitable substrate, delivering the isoxazoline $(3 \mathrm{~m})$ was useful yield. Then, oximes with substituted allylic group were tested. Oxime with double-methyl substituton at $\mathrm{R}^{1}$ position ( $3 \mathrm{n}, 64 \%$ yield) showed higher reactivity than unsubstituted one (3f, $48 \%$ yield). Moreover, oxime with steric 1,1-disubstituted alkene group was converted smoothly to the corresponding product (30,60\% yield). Finally, oxime of phenyl cyclohex-2-enyl methanone, having a cyclic allyl unit also proved to be a reactive substrate, transforming into theproduct $3 p$ in $74 \%$ yield.
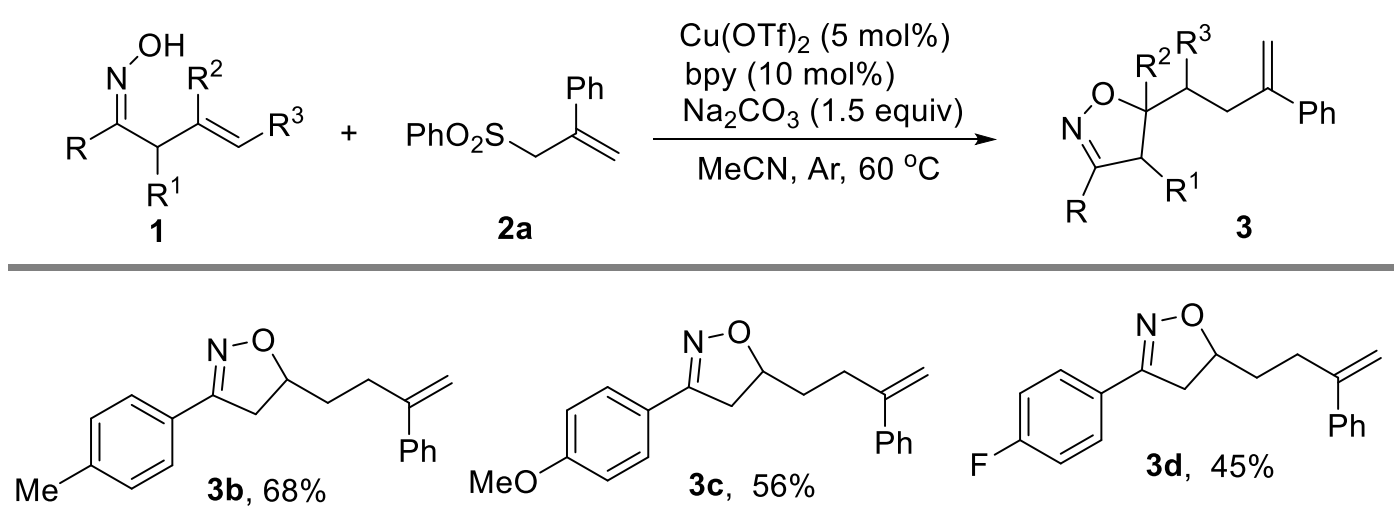<smiles>C=C(CCC1CC(c2ccc(C(=O)OC(C)(C)C)cc2)=NO1)c1ccc(C(=O)c2ccccc2)cc1</smiles><smiles>C=C(CCC1CC(c2ccc([N+](=O)[O-])cc2)=NO1)c1ccccc1</smiles><smiles>C=C(CCC1CC(c2cccs2)=NO1)c1ccccc1</smiles><smiles>C=C(CCC1ON=C(c2ccc(Br)cc2)C1(C)C)c1ccccc1</smiles>

3n $64 \%$<smiles>C=C(CCC1(C)CC(c2ccccc2)=NO1)c1ccccc1</smiles>

$30,60 \%$

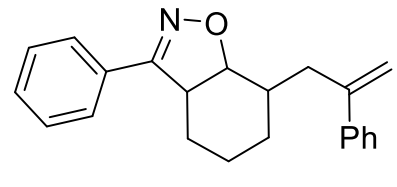

$3 p, 74 \%, d r=3.2: 1$

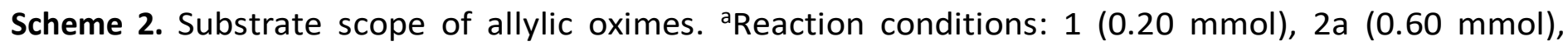
$\mathrm{Cu}(\mathrm{OTf})_{2}(5 \mathrm{~mol} \%)$, bpy $(10 \mathrm{~mol} \%)$ and $\mathrm{Na}_{2} \mathrm{CO}_{3}(0.30 \mathrm{mmol})$ in $\mathrm{MeCN}(2.0 \mathrm{~mL})$ at $60{ }^{\circ} \mathrm{C}$ for $12 \mathrm{~h}$, under argon. ' Isolated yields. 
Next, we surveyed various allyl sulfones. As summarized in Scheme 3, when the phenyl group in 1a was replaced by ester group, isoxazoline with useful $\alpha, 6$-unsaturated ester groups 4a-c were prepared in 51-62\% yields. Similarly, cyano and benzoyl substituted allyl sulfones were successfully engaged in the cyclization to yield products $\mathbf{4 d - e}$ in $52 \%$ and $32 \%$ yields, respectively.

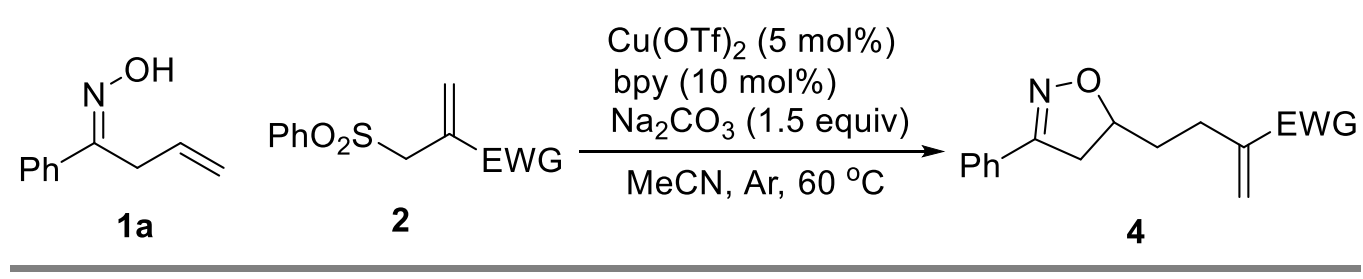<smiles>C=C(CCC1CC(c2ccccc2)=NO1)COC</smiles>

$4 a, 56 \%$<smiles>C=C(CCC1CC(c2ccccc2)=NO1)C(=O)OCC</smiles>

$4 b, 62 \%$<smiles>C=C(CCC1CC(c2ccccc2)=NO1)C(=O)OCC</smiles>

4c, $51 \%$<smiles>C=C(C#N)CCC1CC(c2ccccc2)=NO1</smiles>

$4 d, 52 \%$<smiles>C=C(CCC1CC(c2ccccc2)=NO1)C(=O)c1ccccc1</smiles>

$4 \mathbf{e}, 32 \%$

Scheme 3. Substrate scope of allyl sulfones. ${ }^{a}$ Reaction conditions: $1 \mathrm{a}(0.20 \mathrm{mmol}), 2 \mathrm{a}(0.60 \mathrm{mmol})$, $\mathrm{Cu}(\mathrm{OTf})_{2}(5 \mathrm{~mol} \%)$, bpy $(10 \mathrm{~mol} \%)$ and $\mathrm{Na}_{2} \mathrm{CO}_{3}(0.30 \mathrm{mmol})$ in $\mathrm{MeCN}(2 \mathrm{~mL})$ at $60{ }^{\circ} \mathrm{C}$ for $12 \mathrm{~h}$, under argon. 'Isolated yields.

To show practicality of this protocol, we scaled up the reaction to $3 \mathrm{mmol}$, and the isoxazoline $3 a$ was isolated in $52 \%$ yield $(0.43 \mathrm{~g}$ ) (Scheme $4 \mathrm{a})$. The synthetic utility of the alkene-containing isoxazoline was exemplified by the oxidative cleavage of $\mathrm{C}=\mathrm{C}$ double bond ${ }^{44}$ into isoxazoline with ketone side chain. Treatment of 3a with 2 equiv of oxone and 1.5 equiv of $\mathrm{NaIO}_{4}$ under $80{ }^{\circ} \mathrm{C}$ led to the isolation of $5 \mathrm{a}$ in $50 \%$ yield (Scheme 4b).

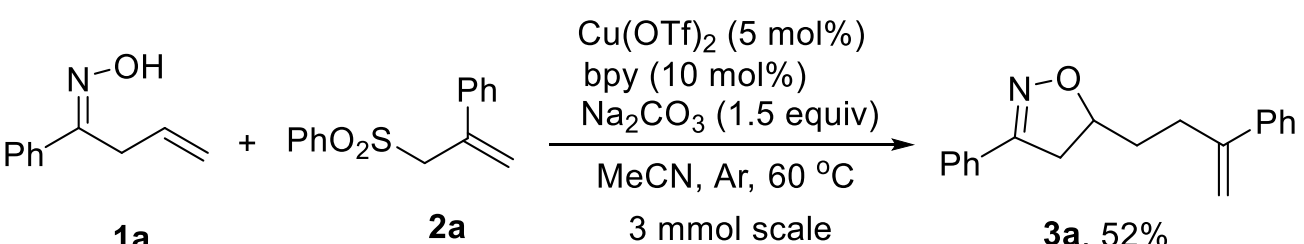

$1 \mathbf{a}$

$3 \mathrm{mmol}$ scale

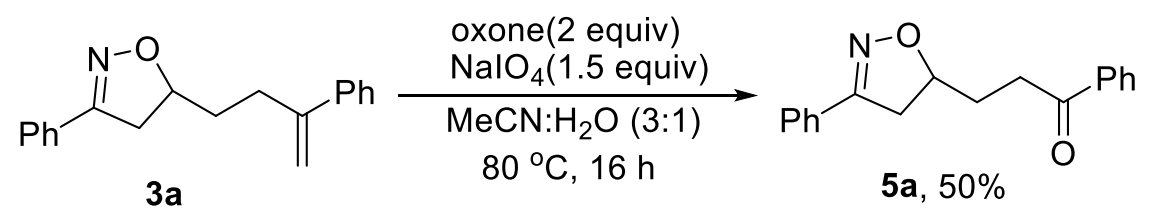

Scheme 4. Gram scale reaction and product derivatization.

According to the above experimental results and previous reports, a plausible mechanism is proposed (Scheme 5). Firstly, iminoxyl anion A, formed by deprotonation of 
substrates 1 were SET oxidized by $\mathrm{Cu}(\mathrm{II})$, to generate the iminoxyl radical $\mathrm{B} .{ }^{32}$ Then, iminoxyl radical $\mathbf{B}$ undergoes 5-exo-trig radical cyclization, delivering the carbon-centered radical intermediate $\mathbf{C}$. The addition of the radical $\mathbf{C}$ onto the $\mathrm{C}=\mathrm{C}$ bond of allyl sulfone $\mathbf{2}$ followed by desulfonation leads to the product 3 and a sulfonyl radical. ${ }^{45,46}$ Finally, SET reduction of sulfonyl radical by $\mathrm{Cu}(\mathrm{I})$ forms the benzenesulfinic acid anion with the concomitant regeneration of $\mathrm{Cu}(\mathrm{II})$.

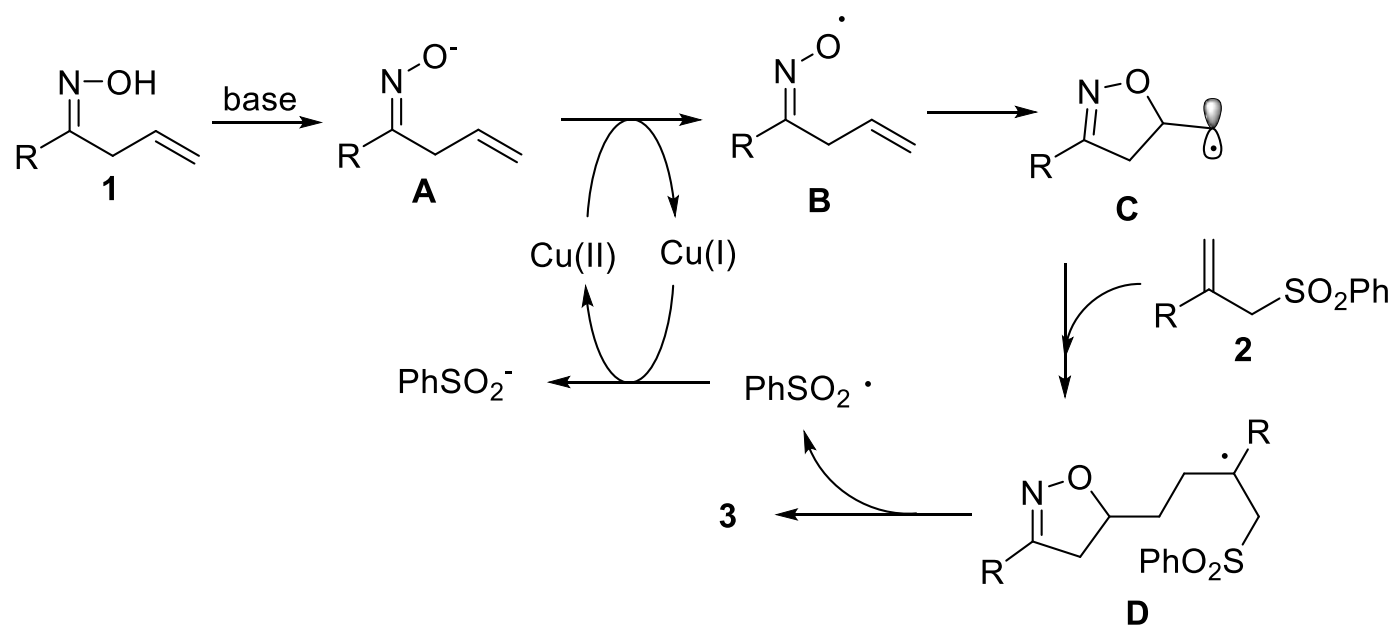

Scheme 5. Proposed mechanism.

\section{Conclusions}

We have developed an efficient copper-catalyzed radical oxyallylation of alkenes with allylic sulfones using oximes as the oxygen-centered radical precursor. This new method provided a practical approach to isoxazolines containing an alkene moiety in moderate to good yields, which may act as a versatile platform allowing further downstream diversification.

\section{Experimental Section}

General. Unless stated otherwise, all reactions were carried out under an argon atmosphere. All commercial reagents were used without additional purification. Flash chromatography was carried out with silica gel (200300 mesh). Melting points were determined without correction on a digital melting-point apparatus. ${ }^{1} \mathrm{H} N M R$, ${ }^{13} \mathrm{C}$ NMR and ${ }^{19} \mathrm{~F}$ NMR spectra were recorded at 500 (600) MHz, 125 (150) MHz and $400 \mathrm{MHz}$ spectrometers in $\mathrm{CDCl}_{3}$ using tetramethylsilane (TMS) as internal standard, respectively. High resolution mass spectra (HRMS) were obtained by the ESI ionization sources.

Experimental procedures and characterization of products. To a dried reaction tube was added allyl oxime 1 $(0.20 \mathrm{mmol}), 2(0.60 \mathrm{mmol}), \mathrm{Cu}(\mathrm{OTf})_{2}(5 \mathrm{~mol} \%)$, bpy $(10 \mathrm{~mol} \%), \mathrm{Na}_{2} \mathrm{CO}_{3}(0.30 \mathrm{mmol})$. The tube was evacuated and backfilled with argon (three times). Then, anhydrous MeCN (2.0 mL) was injected into the tube by syringe. The solution was kept at $60{ }^{\circ} \mathrm{C}$ for $12 \mathrm{~h}$. The reaction mixture was partitioned with EtOAc and water. The organic layer was separated and washed with brine, dried over anhydrous $\mathrm{Na}_{2} \mathrm{SO}_{4}$ and concentrated under 
reduced pressure. The crude mixture was purified by silica gel column chromatography (PE/EA 30:1, v/v) to give the corresponding product 3 or 4.

3-Phenyl-5-(3-phenylbut-3-en-1-yl)-4,5-dihydroisoxazole (3a). Colorless liquid (80\%, $48 \mathrm{mg}), \mathrm{R}_{\mathrm{f}} 0.49$ (petroleum ether/ethyl acetate 10:1). ${ }^{1} \mathrm{H}$ NMR (500 MHz, $\left.\mathrm{CDCl}_{3}\right) \delta$ 7.60-7.56 (m, $\left.2 \mathrm{H}\right), 7.42(\mathrm{~m}, 2 \mathrm{H}), 7.39-7.32$ $(\mathrm{m}, 4 \mathrm{H}), 7.30-7.26(\mathrm{~m}, 1 \mathrm{H}), 5.33(\mathrm{~d}, J 0.8 \mathrm{~Hz}, 1 \mathrm{H}), 5.13(\mathrm{~d}, J 1.2 \mathrm{~Hz}, 1 \mathrm{H}), 4.78(\mathrm{dtd}, J$ 10.4, 7.9, $5.3 \mathrm{~Hz}, 1 \mathrm{H})$, 3.36 (dd, J 16.4, 10.4 Hz, $1 \mathrm{H}), 2.92(\mathrm{dd}, J$ 16.4, $8.1 \mathrm{~Hz}, 1 \mathrm{H}), 2.77(\mathrm{~m}, 1 \mathrm{H}), 2.65(\mathrm{~m}, 1 \mathrm{H}), 2.00-1.85(\mathrm{~m}, 1 \mathrm{H})$, 1.87-1.70 (m, $1 \mathrm{H}) .{ }^{13} \mathrm{C} \mathrm{NMR}\left(125 \mathrm{MHz}, \mathrm{CDCl}_{3}\right) \delta 156.4,147.4,140.7,129.9,128.7,128.4,127.6,126.6,126.1$, 113.0, 80.7, 77.3, 77., 76.8, 40.0, 34.1, 31.3. HRMS (ESI): $\mathrm{m} / \mathrm{z}$ calcd for $\mathrm{C}_{19} \mathrm{H}_{20} \mathrm{NO}^{+}(\mathrm{M}+\mathrm{H})^{+} 278.1539$, found 278.1548.

4-Phenyl-5-(3-phenylbut-3-en-1-yl)-4,5-dihydroisoxazole (3b). White solid (40 mg, 68\%), mp $45-47{ }^{\circ} \mathrm{C}, \mathrm{R}_{\mathrm{f}} 0.53$ (petroleum ether/ethyl acetate 10:1). ${ }^{1} \mathrm{H} N M R\left(500 \mathrm{MHz}, \mathrm{CDCl}_{3}\right) \delta 7.55(\mathrm{~d}, J 8.2 \mathrm{~Hz}, 2 \mathrm{H}), 7.44(\mathrm{~d}, J 7.5 \mathrm{~Hz}, 2 \mathrm{H})$, 7.35 (dd, J 10.2, 4.8 Hz, 2 H), $7.29(\mathrm{~m}, 1 \mathrm{H}), 7.20$ (d, J $8.0 \mathrm{~Hz}, 2 \mathrm{H}), 5.33(\mathrm{~s}, 1 \mathrm{H}), 5.14$ (d, J $1.1 \mathrm{~Hz}, 1 \mathrm{H}), 4.75$ (dtd, J 10.3, 7.8, 5.3 Hz, $1 \mathrm{H}$ ), 3.38 (dd, J 16.4, 10.3 Hz, $1 \mathrm{H}), 2.94$ (dd, J 16.4, 8.0 Hz, $1 \mathrm{H}), 2.77(\mathrm{~m}, 1 \mathrm{H}), 2.71-2.59(\mathrm{~m}$, $1 \mathrm{H}), 2.38(\mathrm{~s}, 3 \mathrm{H}), 1.99-1.90(\mathrm{~m}, 1 \mathrm{H}), 1.83-1.74(\mathrm{~m}, 1 \mathrm{H}) .{ }^{13} \mathrm{C} \mathrm{NMR}\left(125 \mathrm{MHz}, \mathrm{CDCl}_{3}\right) \delta$ 156.4, 147.4, 140.8, 140.1, 129.4, 128.4, 127.5, 127.0, 126.5, 126.1, 112.9, 80.5, 77.3, 77.0, 76.8, 40.1, 34.0, 31.3, 21.4. HRMS (ESI): $\mathrm{m} / \mathrm{z}$ calcd for $\mathrm{C}_{20} \mathrm{H}_{21} \mathrm{NONa}^{+}(\mathrm{M}+\mathrm{Na})^{+} 314.1515$, found 314.1517 .

3-(4-Methoxyphenyl)-5-(3-phenylbut-3-en-1-yl)-4,5-dihydroisoxazole (3c). White solid (36 mg, 56\%), $\mathrm{mp} \mathrm{72-}$ $74{ }^{\circ} \mathrm{C}, \mathrm{R}_{\mathrm{f}} 0.32$ (petroleum ether/ethyl acetate 10:1). ${ }^{1} \mathrm{H} \mathrm{NMR}\left(500 \mathrm{MHz}, \mathrm{CDCl}_{3}\right) \delta$ 7.63-7.54 (m, $\left.2 \mathrm{H}\right), 7.43(\mathrm{~m}, 2$ H), 7.36-7.31 (m, $2 \mathrm{H}), 7.28(\mathrm{~m}, 1 \mathrm{H}), 6.93-6.89(\mathrm{~m}, 2 \mathrm{H}), 5.32(\mathrm{~d}, J 0.9 \mathrm{~Hz}, 1 \mathrm{H}), 5.13(\mathrm{~d}, J 1.2 \mathrm{~Hz}, 1 \mathrm{H}), 4.73(\mathrm{dtd}$, J 10.3, 7.8, 5.3 Hz, $1 \mathrm{H}), 3.83(\mathrm{~s}, 3 \mathrm{H}), 3.36$ (dd, J 16.3, 10.3 Hz, $1 \mathrm{H}), 2.93(\mathrm{dd}, J$ 16.4, $8.0 \mathrm{~Hz}, 1 \mathrm{H}), 2.76(\mathrm{~m}, 1 \mathrm{H})$, $2.65(\mathrm{~m}, 1 \mathrm{H}), 2.00-1.86(\mathrm{~m}, 1 \mathrm{H}), 1.84-1.70(\mathrm{~m}, 1 \mathrm{H}) .{ }^{13} \mathrm{C} \mathrm{NMR}\left(125 \mathrm{MHz}, \mathrm{CDCl}_{3}\right) \delta 161.0,156.0,147.4,140.8$, 128.4, 128.1, 127.5, 126.1, 122.4, 114.1, 112.9, 80.4, 77.3, 77.0, 76.8, 55.3, 40.2, 34.0, 31.4. HRMS (ESI): $\mathrm{m} / \mathrm{z}$ calcd for $\mathrm{C}_{20} \mathrm{H}_{21} \mathrm{NO}_{2} \mathrm{Na}^{+}(\mathrm{M}+\mathrm{Na})^{+} 330.1470$, found 330.1469

3-(4-Fluorophenyl)-5-(3-phenylbut-3-en-1-yl)-4,5-dihydroisoxazole (3d). White solid (26 mg, 45\%), mp 79-80 ${ }^{\circ} \mathrm{C}, \mathrm{R}_{\mathrm{f}} 0.41$ (petroleum ether/ethyl acetate 10:1). ${ }^{1} \mathrm{H}$ NMR $\left(500 \mathrm{MHz}, \mathrm{CDCl}_{3}\right) \delta$ 7.55-7.49 (m, $\left.4 \mathrm{H}\right), 7.44-7.40(\mathrm{~m}$, $2 \mathrm{H}), 7.37-7.31(\mathrm{~m}, 2 \mathrm{H}), 7.30-7.26(\mathrm{~m}, 1 \mathrm{H}), 5.33(\mathrm{~d}, J 0.9 \mathrm{~Hz}, 1 \mathrm{H}), 5.13(\mathrm{~d}, J 1.2 \mathrm{~Hz}, 1 \mathrm{H}), 4.78(\mathrm{dtd}, J$ 10.4, 7.9, $5.3 \mathrm{~Hz}, 1 \mathrm{H}), 3.36(\mathrm{dd}, J 16.4,10.4 \mathrm{~Hz}, 1 \mathrm{H}), 2.92(\mathrm{dd}, J 16.5,8.1 \mathrm{~Hz}, 1 \mathrm{H}), 2.76(\mathrm{~m}, 1 \mathrm{H}), 2.65(\mathrm{~m}, 1 \mathrm{H}), 2.01-1.85$ $(\mathrm{m}, 1 \mathrm{H}), 1.86-1.70(\mathrm{~m}, 1 \mathrm{H}) .{ }^{13} \mathrm{C} \mathrm{NMR}\left(125 \mathrm{MHz}, \mathrm{CDCl}_{3}\right) \delta 163.7$ (d, J 251.8 Hz), 155.5, 147.3, 140.7, 128.5(d, J 8.3 Hz), 128.4, 127.6, 126.1, 115.9 (d, J 21.4 Hz), 113.0, 80.8, 77.3, 77.0, 76.8, 40.0, 34.0, 31.3. ${ }^{19} \mathrm{~F} \mathrm{NMR} \mathrm{(400}$ $\left.\mathrm{MHz}, \mathrm{CDCl}_{3}\right) \delta$ 112.7. HRMS (ESI): $\mathrm{m} / z$ calcd for $\mathrm{C}_{19} \mathrm{H}_{18} \mathrm{FNONa}^{+}(\mathrm{M}+\mathrm{Na})^{+} 318.1265$, found 318.1267.

3-(4-Chlorophenyl)-5-(3-phenylbut-3-en-1-yl)-4,5-dihydroisoxazole (3e). White solid (36 mg, 57\%), mp 82-84 ${ }^{\circ} \mathrm{C}, \mathrm{R}_{\mathrm{f}} 0.54$ (petroleum ether/ethyl acetate 10:1). ${ }^{1} \mathrm{H} \mathrm{NMR}\left(500 \mathrm{MHz}, \mathrm{CDCl}_{3}\right) \delta$ 7.60-7.56 (m, $\left.2 \mathrm{H}\right), 7.42(\mathrm{~m}, 2 \mathrm{H})$, 7.39-7.31 (m, $4 \mathrm{H}), 7.30-7.26(\mathrm{~m}, 1 \mathrm{H}), 5.33(\mathrm{~d}, J 0.8 \mathrm{~Hz}, 1 \mathrm{H}), 5.13(\mathrm{~d}, J 1.2 \mathrm{~Hz}, 1 \mathrm{H}), 4.78(\mathrm{dtd}, J$ 10.4, 7.9, 5.3 Hz, $1 \mathrm{H}), 3.36$ (dd, J 16.4, $10.4 \mathrm{~Hz}, 1 \mathrm{H}), 2.92(\mathrm{dd}, J 16.4,8.1 \mathrm{~Hz}, 1 \mathrm{H}), 2.77(\mathrm{~m}, 1 \mathrm{H}), 2.65(\mathrm{~m}, 1 \mathrm{H}), 2.00-1.89(\mathrm{~m}, 1$ $\mathrm{H}), 1.85-1.71(\mathrm{~m}, 1 \mathrm{H}) .{ }^{13} \mathrm{C} \mathrm{NMR}\left(125 \mathrm{MHz}, \mathrm{CDCl}_{3}\right) \delta 155.5,147.3,140.7,135.9,128.9,128.4,127.8,127.6$, 126.1, 113.0, 81.0, 77.3, 77.0, 76.8, 39.8, 34.0, 31.3. HRMS (ESI): $\mathrm{m} / z$ calcd for $\mathrm{C}_{19} \mathrm{H}_{18} \mathrm{ClNONa}^{+}(\mathrm{M}+\mathrm{Na})^{+}$ 334.0975, found 334.0975.

3-(4-Bromophenyl)-5-(3-phenylbut-3-en-1-yl)-4,5-dihydroisoxazole (3f). White solid (34 mg, 48\%), mp 94-95 ${ }^{\circ} \mathrm{C}, \mathrm{R}_{\mathrm{f}} 0.45$ (petroleum ether/ethyl acetate 10:1). ${ }^{1} \mathrm{H}$ NMR $\left(500 \mathrm{MHz}, \mathrm{CDCl}_{3}\right) \delta$ 7.55-7.49 (m, $\left.4 \mathrm{H}\right), 7.44-7.40(\mathrm{~m}$, $2 \mathrm{H}), 7.37-7.31(\mathrm{~m}, 2 \mathrm{H}), 7.30-7.26(\mathrm{~m}, 1 \mathrm{H}), 5.33(\mathrm{~d}, J 0.9 \mathrm{~Hz}, 1 \mathrm{H}), 5.13(\mathrm{~d}, J 1.2 \mathrm{~Hz}, 1 \mathrm{H}), 4.78(\mathrm{dtd}, J$ 10.4, 7.9, $5.3 \mathrm{~Hz}, 1 \mathrm{H}), 3.36(\mathrm{dd}, J 16.4,10.4 \mathrm{~Hz}, 1 \mathrm{H}), 2.92(\mathrm{dd}, J 16.5,8.1 \mathrm{~Hz}, 1 \mathrm{H}), 2.76(\mathrm{~m}, 1 \mathrm{H}), 2.65(\mathrm{~m}, 1 \mathrm{H}), 2.01-1.85$ $(\mathrm{m}, 1 \mathrm{H}), 1.86-1.70(\mathrm{~m}, 1 \mathrm{H}) .{ }^{13} \mathrm{C}$ NMR $\left(125 \mathrm{MHz}, \mathrm{CDCl}_{3}\right) \delta 155.6,147.3,140.7,131.9,128.8,128.4,128.0,127.6$, $126.1,124.2,113.0,81.0,77.3,77.0,76.8,39.8,34.0,31.3$. HRMS (ESI): $m / z$ calcd for $\mathrm{C}_{19} \mathrm{H}_{18} \mathrm{BrNONa}^{+}(\mathrm{M}+\mathrm{Na})^{+}$ 378.0464 , found 378.0465 . 
5-(3-Phenylbut-3-en-1-yl)-3-(4-(trifluoromethyl)phenyl)-4,5-dihydroisoxazole (3g). White solid (32 mg, 46\%), mp 99-100 ${ }^{\circ} \mathrm{C}, \mathrm{R}_{\mathrm{f}} 0.46$ (petroleum ether/ethyl acetate 10:1). ${ }^{1} \mathrm{H} \mathrm{NMR}\left(500 \mathrm{MHz}, \mathrm{CDCl}_{3}\right) \delta 7.78(\mathrm{~d}, J 8.1 \mathrm{~Hz}, 2 \mathrm{H})$, 7.67 (d, J 8.3 Hz, 2 H), 7.63-7.26 (m, 5 H), 5.35 (d, J 1.0 Hz, 1 H), 5.16 (d, J 1.2 Hz, 1 H), 4.82 (dtd, J 10.5, 7.9, 5.3 Hz, 1 H)., 3.40 (dd, J 16.5, 10.5 Hz, 1 H), 2.96 (dd, J 16.5, 8.1 Hz, 1 H)., 2.78 (m, 1 H), 2.67(m, 1 H), $2.04-1.92(m$, $1 \mathrm{H}), 1.88-1.76(\mathrm{~m}, 1 \mathrm{H}) .{ }^{13} \mathrm{C} \mathrm{NMR}\left(125 \mathrm{MHz}, \mathrm{CDCl}_{3}\right) \delta 155.4,147.2,140.6,133.2,131.7(\mathrm{~d}, J 1.0 \mathrm{~Hz})(\mathrm{q}, J 32.8$ $\mathrm{Hz}), 128.4,127.6,126.8,126.1,125.6$ (q, J $3.7 \mathrm{~Hz}), 124.9$ (q, J 272.9 Hz), 113.1, 81.4, 77.3, 77.0, 76.8, 39.6, 34.01, 31.3. $\left.{ }^{19} \mathrm{~F} \mathrm{NMR} \mathrm{(400} \mathrm{MHz,} \mathrm{CDCl} 3\right) \delta 63.9$. HRMS (ESI): $\mathrm{m} / z$ calcd for $\mathrm{C}_{20} \mathrm{H}_{18} \mathrm{~F}_{3} \mathrm{NONa}^{+}(\mathrm{M}+\mathrm{Na})^{+} 368.1238$, found 368.1231.

3-(4-Nitrophenyl)-5-(3-phenylbut-3-en-1-yl)-4,5-dihydroisoxazole (3h). Yellow solid (30 mg, 47\%), mp 73-75 ${ }^{\circ} \mathrm{C}, \mathrm{R}_{\mathrm{f}} 0.29$ (petroleum ether/ethyl acetate 10:1). ${ }^{1} \mathrm{H}$ NMR $\left(500 \mathrm{MHz}, \mathrm{CDCl}_{3}\right) \delta 8.28-8.21(\mathrm{~m}, 2 \mathrm{H}), 7.85-7.75(\mathrm{~m}$, $2 \mathrm{H}), 7.46-7.38(\mathrm{~m}, 2 \mathrm{H}), 7.37-7.32(\mathrm{~m}, 2 \mathrm{H}), 7.31-7.26(\mathrm{~m}, 1 \mathrm{H}), 5.34(\mathrm{~d}, J 1.1 \mathrm{~Hz}, 1 \mathrm{H}), 5.14(\mathrm{~d}, J 1.2 \mathrm{~Hz}, 1 \mathrm{H})$, 4.86 (dtd, J 10.6, 8.0, 5.3 Hz, $1 \mathrm{H}$ ), 3.41 (dd, J 16.5, 10.6 Hz, $1 \mathrm{H}$ ), 2.97 (dd, J 16.5, 8.2 Hz, $1 \mathrm{H}$ ), $2.83-2.72$ (m, 1 $\mathrm{H}), 2.71-2.61(\mathrm{~m}, 1 \mathrm{H}), 2.02-1.90(\mathrm{~m}, 1 \mathrm{H}), 1.89-1.76(\mathrm{~m}, 1 \mathrm{H}) .{ }^{13} \mathrm{C} \mathrm{NMR}\left(125 \mathrm{MHz}, \mathrm{CDCl}_{3}\right) \delta 154.9,148.4,147.1$, 140.6, 135.9, 128.5, 127.6, 127.2, 126.1, 124.0, 113.2, 82.0, 77.3, 77.0, 76.8, 39.4, 34.0, 31.2. HRMS (ESI): $\mathrm{m} / \mathrm{z}$ calcd for $\mathrm{C}_{19} \mathrm{H}_{19} \mathrm{~N}_{2} \mathrm{O}_{3}{ }^{+}(\mathrm{M}+\mathrm{H})^{+} 323.1390$, found 323.1391 .

Methyl-4-(5-(3-phenylbut-3-en-1-yl)-4,5-dihydroisoxazol-3-yl)benzoate (3i). White solid (18 mg, 37\%), mp 103-105 ${ }^{\circ} \mathrm{C}, \mathrm{R}_{\mathrm{f}} 0.28$ (petroleum ether/ethyl acetate 10:1). ${ }^{1} \mathrm{H}$ NMR $\left(500 \mathrm{MHz}, \mathrm{CDCl}_{3}\right) \delta 8.09-8.03(\mathrm{~m}, 2 \mathrm{H}), 7.73-$ $7.69(\mathrm{~m}, 2 \mathrm{H}), 7.42(\mathrm{~m}, 2 \mathrm{H}), 7.37-7.31(\mathrm{~m}, 2 \mathrm{H}), 7.28(\mathrm{~m}, 1 \mathrm{H}), 5.33(\mathrm{~d}, J 1.0 \mathrm{~Hz}, 1 \mathrm{H}), 5.13(\mathrm{~d}, J 1.2 \mathrm{~Hz}, 1 \mathrm{H}), 4.81$ (dtd, J 10.5, 7.9, $5.4 \mathrm{~Hz}, 1 \mathrm{H}), 3.93(\mathrm{~s}, 3 \mathrm{H}), 3.40$ (dd, J 16.5, $10.5 \mathrm{~Hz}, 1 \mathrm{H}), 2.96$ (dd, J 16.5, 8.1 Hz, $1 \mathrm{H}), 2.77(\mathrm{~m}$, $1 \mathrm{H}), 2.66(\mathrm{~m}, 1 \mathrm{H}), 2.01-1.88(\mathrm{~m}, 1 \mathrm{H}), 1.87-1.73(\mathrm{~m}, 1 \mathrm{H}) .{ }^{13} \mathrm{C} \mathrm{NMR}\left(125 \mathrm{MHz}, \mathrm{CDCl}_{3}\right) \delta 166.5,155.8,147.3,140.7$, 134.0, 131.2, 129.9, 128.4, 127.6, 126.4, 126.1, 113.1, 81.3, 77.3, 77.0, 76.8, 52.3, 39.6, 34.0, 31.3. HRMS (ESI): $\mathrm{m} / z$ calcd for $\mathrm{C}_{21} \mathrm{H}_{21} \mathrm{NO}_{3} \mathrm{Na}^{+}(\mathrm{M}+\mathrm{Na})^{+} 358.1419$, found 358.1434 .

3-(3-Nitrophenyl)-5-(3-phenylbut-3-en-1-yl)-4,5-dihydroisoxazole (3j). Pale yellow oil (16 mg, $40 \%), R_{f} 0.27$ (petroleum ether/ethyl acetate 10:1). ${ }^{1} \mathrm{H} \mathrm{NMR}\left(500 \mathrm{MHz}, \mathrm{CDCl}_{3}\right) \delta 8.39(\mathrm{t}, J 1.9 \mathrm{~Hz}, 1 \mathrm{H}), 8.25(\mathrm{~m}, 1 \mathrm{H}), 8.10-$ $8.04(\mathrm{~m}, 1 \mathrm{H}), 7.59(\mathrm{t}, J 8.0 \mathrm{~Hz}, 1 \mathrm{H}), 7.46-7.40(\mathrm{~m}, 2 \mathrm{H}), 7.37-7.32(\mathrm{~m}, 2 \mathrm{H}), 7.30-7.26(\mathrm{~m}, 1 \mathrm{H}), 5.33(\mathrm{~d}, J 1.0 \mathrm{~Hz}$, $1 \mathrm{H}), 5.14$ (d, J $1.2 \mathrm{~Hz}, 1 \mathrm{H}), 4.86$ (dtd, J 10.5, 7.9, $5.4 \mathrm{~Hz}, 1 \mathrm{H}$ ), 3.43 (dd, J 16.5, $10.5 \mathrm{~Hz}, 1 \mathrm{H}$ ), 3.00 (dd, J 16.5, $8.1 \mathrm{~Hz}, 1 \mathrm{H}), 2.82-2.73(\mathrm{~m}, 1 \mathrm{H}), 2.67(\mathrm{~m}, 1 \mathrm{H}), 2.02-1.92(\mathrm{~m}, 1 \mathrm{H}), 1.87-1.76(\mathrm{~m}, 1 \mathrm{H}) .{ }^{13} \mathrm{C} \mathrm{NMR}\left(125 \mathrm{MHz}, \mathrm{CDCl}_{3}\right)$ $\delta 154.8,148.5,147.2,140.6,132.1,131.69,129.8,128.5,127.6,126.1,124.4,121.4,113.2,81.7,77.3,77.0$, 76.8, 39.5, 34.0, 31.3. HRMS (ESI): $m / z$ calcd for $\mathrm{C}_{19} \mathrm{H}_{18} \mathrm{~N}_{2} \mathrm{O}_{3} \mathrm{Na}^{+}(\mathrm{M}+\mathrm{Na})^{+} 345.1215$, found 345.1212.

5-(3-Phenylbut-3-en-1-yl)-3-(thiophen-2-yl)-4,5-dihydroisoxazole (3k). Brown oil (27mg, 50\%), $\mathrm{R}_{\mathrm{f}} 0.38$ (petroleum ether/ethyl acetate 10:1). ${ }^{1} \mathrm{H}$ NMR $\left(500 \mathrm{MHz}, \mathrm{CDCl}_{3}\right) \delta 7.44-7.39(\mathrm{~m}, 2 \mathrm{H}), 7.39-7.30(\mathrm{~m}, 3 \mathrm{H}), 7.28$ $(d, J 7.4 \mathrm{~Hz}, 1 \mathrm{H}), 7.16(\mathrm{~d}, J 3.6 \mathrm{~Hz}, 1 \mathrm{H}), 7.05(\mathrm{dd}, J 5.0,3.7 \mathrm{~Hz}, 1 \mathrm{H}), 5.32(\mathrm{~s}, 1 \mathrm{H}), 5.13(\mathrm{~d}, J 1.0 \mathrm{~Hz}, 1 \mathrm{H}), 4.76$ (dtd, J 10.3, 7.8, 5.4 Hz, $1 \mathrm{H}$ ), 3.40 (dd, J 16.3, 10.3 Hz, $1 \mathrm{H}), 2.96(\mathrm{dd}, J 16.3,8.0 \mathrm{~Hz}, 1 \mathrm{H}), 2.76(\mathrm{~m}, 1 \mathrm{H}), 2.69-$ $2.58(\mathrm{~m}, 1 \mathrm{H}), 2.05-1.86(\mathrm{~m}, 1 \mathrm{H}), 1.83-1.70(\mathrm{~m}, 1 \mathrm{H}) .{ }^{13} \mathrm{C} \mathrm{NMR}\left(125 \mathrm{MHz}, \mathrm{CDCl}_{3}\right) \delta 128.4,128.1,127.6,127.2$, 126.1, 113.0, 81.0, 77.3, 77.0, 76.8, 40.8, 33.9, 31.3. HRMS (ESI): $\mathrm{m} / z$ calcd for $\mathrm{C}_{17} \mathrm{H}_{17} \mathrm{NOSNa}^{+}\left(\mathrm{M}+\mathrm{Na}^{+}\right.$ 306.0923, found 306.0924.

3-(Naphthalen-2-yl)-5-(3-phenylbut-3-en-1-yl)-4,5-dihydroisoxazole (3l). White solid (30 mg, 51\%), mp 114$114{ }^{\circ} \mathrm{C}, \mathrm{R}_{\mathrm{f}} 0.46$ (petroleum ether/ethyl acetate 10:1). ${ }^{1} \mathrm{H} \mathrm{NMR}\left(500 \mathrm{MHz}, \mathrm{CDCl}_{3}\right) \delta 7.98(\mathrm{dd}, J 8.7,1.6 \mathrm{~Hz}, 1 \mathrm{H})$, 7.85 (m, 4 H), 7.57-7.47 (m, 2 H), 7.47-7.41 (m, 2 H), 7.36 (dd, J 10.2, 4.8 Hz, 2 H), 7.32-7.27 (m, 1 H), 5.35 (s, 1 H), 5.16 (d, J $1.1 \mathrm{~Hz}, 1 \mathrm{H}$ ), 4.83 (dtd, J 10.4, 7.8, 5.4 Hz, $1 \mathrm{H}$ ), 3.51 (dd, J 16.3, $10.4 \mathrm{~Hz}, 1 \mathrm{H}$ ), 3.08 (dd, J $16.3,8.0$ $\mathrm{Hz}, 1 \mathrm{H}), 2.80(\mathrm{~m}, 1 \mathrm{H}), 2.73-2.63(\mathrm{~m}, 1 \mathrm{H}), 2.07-1.91(\mathrm{~m}, 1 \mathrm{H}), 1.91-1.76(\mathrm{~m}, 1 \mathrm{H}) .{ }^{13} \mathrm{C} \mathrm{NMR}\left(125 \mathrm{MHz} \mathrm{CDCl}_{3}\right) \delta$ 156.6, 147.4, 140.8, 134.0, 133.0, 128.4, 127.8, 127.5, 127.0, 126.7, 126.1, 123.5, 113.0, 80.9, 77.3, 77.0, 76.77, 39.9, 34.1, 31.3. HRMS (ESI): $m / z$ calcd for $\mathrm{C}_{23} \mathrm{H}_{21} \mathrm{NONa}^{+}(\mathrm{M}+\mathrm{Na})^{+} 350.1521$, found 350.1521. 
3-Phenethyl-5-(3-phenylbut-3-en-1-yl)-4,5-dihydroisoxazole (3m). Pale yellow oil (29 $\mathrm{mg}, 38 \%), \mathrm{R}_{\mathrm{f}} 0.36$ (petroleum ether/ethyl acetate 10:1). ${ }^{1} \mathrm{H}$ NMR $\left(500 \mathrm{MHz}, \mathrm{CDCl}_{3}\right) \delta 7.40(\mathrm{~m}, 2 \mathrm{H}), 7.36-7.32(\mathrm{~m}, 2 \mathrm{H}), 7.31-7.27$ $(\mathrm{m}, 3 \mathrm{H}), 7.21(\mathrm{~m}, 3 \mathrm{H}), 5.31(\mathrm{~d}, J 1.2 \mathrm{~Hz}, 1 \mathrm{H}), 5.09$ (d, J $1.3 \mathrm{~Hz}, 1 \mathrm{H}), 4.54(\mathrm{dtd}, J$ 10.2, 7.7, $5.4 \mathrm{~Hz}, 1 \mathrm{H}), 2.98-$ $2.86(\mathrm{~m}, 3 \mathrm{H}), 2.67(\mathrm{~m}, 3 \mathrm{H}), 2.60-2.51(\mathrm{~m}, 1 \mathrm{H}), 2.47(\mathrm{~m}, 1 \mathrm{H}), 1.81(\mathrm{~m}, 1 \mathrm{H}), 1.67-1.61(\mathrm{~m}, 1 \mathrm{H}) .{ }^{13} \mathrm{C} \mathrm{NMR}(125$ $\left.\mathrm{MHz}_{1} \mathrm{CDCl}_{3}\right) \delta 158.2,1475,140.8,140.6,128.6,128.3,127.5,126.4,126.1,112.8,79.5,77.3,77.0,76.8,42.4$, 33.9, 32.7, 31.3, 29.6. HRMS (ESI): $\mathrm{m} / z$ calcd for $\mathrm{C}_{21} \mathrm{H}_{23} \mathrm{NONa}^{+}(\mathrm{M}+\mathrm{Na})^{+} 328.1672$, found 328.1676.

3-(4-Bromophenyl)-4,4-dimethyl-5-(3-phenylbut-3-en-1-yl)-4,5-dihydroisoxazole (3n). Pink oil (49 mg, 64\%), $R_{\mathrm{f}} 0.55$ (petroleum ether/ethyl acetate 10:1). ${ }^{1} \mathrm{H}$ NMR $\left(500 \mathrm{MHz}, \mathrm{CDCl}_{3}\right) \delta$ 7.55-7.50 (m, $\left.4 \mathrm{H}\right), 7.46-7.43$ (m, 2 H), 7.37-7.33 (m, $2 \mathrm{H}), 7.31-7.27(\mathrm{~m}, 1 \mathrm{H}), 5.35(\mathrm{~s}, 1 \mathrm{H}), 5.17(\mathrm{~d}, J 1.1 \mathrm{~Hz}, 1 \mathrm{H}), 4.14(\mathrm{dd}, J 10.4,2.6 \mathrm{~Hz}, 1 \mathrm{H}), 2.95$ $(\mathrm{m}, 1 \mathrm{H}), 2.68-2.60(\mathrm{~m}, 1 \mathrm{H}), 1.92-1.82(\mathrm{~m}, 1 \mathrm{H}), 1.72-1.63(\mathrm{~m}, 1 \mathrm{H}), 1.28(\mathrm{~s}, 3 \mathrm{H}), 1.15(\mathrm{~s}, 3 \mathrm{H}) .{ }^{13} \mathrm{C} \mathrm{NMR}(125$ $\left.\mathrm{MHz}_{\mathrm{CDCl}}\right) \delta 164.3,147.6,140.7,131.8,128.8,128.6,128.4,127.5,126.1,124.0,113.1,90.29,77.3,77.0$, 76.8, 50.6, 32.2, 26.8, 24.0, 19.5. HRMS (ESI): $\mathrm{m} / \mathrm{z}$ calcd for $\mathrm{C}_{21} \mathrm{H}_{22} \mathrm{BrNONa}^{+}(\mathrm{M}+\mathrm{Na})^{+} 406.0777$, found 406.0780 .

5-Methyl-3-phenyl-5-(3-phenylbut-3-en-1-yl)-4,5-dihydroisoxazole (3o). Colorless oil (35 mg, 60\%), $\mathrm{R}_{\mathrm{f}} 0.54$ (petroleum ether/ethyl acetate 10:1). ${ }^{1} \mathrm{H}$ NMR $\left(600 \mathrm{MHz}, \mathrm{CDCl}_{3}\right) \delta$ 7.69-7.64 (m, $\left.2 \mathrm{H}\right), 7.45-7.38(\mathrm{~m}, 5 \mathrm{H}), 7.37-$ $7.31(\mathrm{~m}, 2 \mathrm{H}), 7.30-7.25(\mathrm{~m}, 1 \mathrm{H}), 5.30(\mathrm{~d}, J 0.9 \mathrm{~Hz}, 1 \mathrm{H}), 5.12(\mathrm{~d}, J 1.2 \mathrm{~Hz}, 1 \mathrm{H}), 3.19(\mathrm{~d}, J 16.5 \mathrm{~Hz}, 1 \mathrm{H}), 3.06(\mathrm{~d}, J$ $16.5 \mathrm{~Hz}, 1 \mathrm{H}), 2.71-2.62(\mathrm{~m}, 2 \mathrm{H}), 1.95-1.86(\mathrm{~m}, 2 \mathrm{H}), 1.49(\mathrm{~d}, J 7.0 \mathrm{~Hz}, 3 \mathrm{H}) .{ }^{13} \mathrm{C} \mathrm{NMR}\left(150 \mathrm{MHz}, \mathrm{CDCl}_{3}\right) \delta 156.1$, 147.9, 140.9, 130.2, 129.8, 128.7, 128.4, 127.5, 126.5, 126.1, 112.6, 87.0, 77.3, 77.1, 76.9, 45.2, 39.0, 30.1, 25.9. HRMS (ESI) : $m / z$ calcd for $\mathrm{C}_{20} \mathrm{H}_{21} \mathrm{NONa}^{+}(\mathrm{M}+\mathrm{Na})^{+} 314.1515$, found 314.1513.

3-Phenyl-7-(2-phenylallyl)-3a,4,5,6,7,7a-hexahydrobenzo[d]isoxazole (3p). major isomer: Colorless oil (36 $\mathrm{mg}, 56.3 \%), \mathrm{R}_{\mathrm{f}} 0.46$ (petroleum ether/ethyl acetate 10:1). ${ }^{1} \mathrm{H} \mathrm{NMR}\left(600 \mathrm{MHz}, \mathrm{CDCl}_{3}\right) \delta 7.67-7.62(\mathrm{~m}, 2 \mathrm{H}), 7.48$ $7.43(\mathrm{~m}, 2 \mathrm{H}), 7.39$ (dd, J 6.7, 3.6 Hz, $3 \mathrm{H}), 7.35$ (dd, J 10.4, 4.8 Hz, $2 \mathrm{H}), 7.31-7.27(\mathrm{~m}, 1 \mathrm{H}), 5.36(\mathrm{~d}, J 1.3 \mathrm{~Hz}, 1 \mathrm{H})$, $5.10(\mathrm{~d}, J 0.7 \mathrm{~Hz}, 1 \mathrm{H}), 4.34$ (dd, J 8.0, $5.2 \mathrm{~Hz}, 1 \mathrm{H}), 3.46(\mathrm{dd}, J$ 15.9, $7.8 \mathrm{~Hz}, 1 \mathrm{H}), 3.00-2.90(\mathrm{~m}, 1 \mathrm{H}), 2.46(\mathrm{dd}, J$ 14.2, 9.3 Hz, $1 \mathrm{H}), 2.16-2.04(\mathrm{~m}, 1 \mathrm{H}), 1.90(\mathrm{~m}, 1 \mathrm{H}), 1.66(\mathrm{~m}, 1 \mathrm{H}), 1.52-1.42(\mathrm{~m}, 2 \mathrm{H}), 1.35(\mathrm{~m}, 1 \mathrm{H}), 1.30-1.22$ $(\mathrm{m}, 1 \mathrm{H}) .{ }^{13} \mathrm{C}$ NMR $\left(150 \mathrm{MHz}, \mathrm{CDCl}_{3}\right) \delta 162.8,146.2,140.4,129.9,129.4,128.7,128.4,127.6,127.0,126.3,114.4$, 84.7, 77.3, 77.0, 76.8, 44.1, 39.0, 32.9, 24.9, 24.6, 18.7. HRMS (ESI) : $\mathrm{m} / z$ calcd for $\mathrm{C}_{22} \mathrm{H}_{23} \mathrm{NONa}^{+}\left(\mathrm{M}+\mathrm{Na}^{+}\right.$ 340.1672 , found 340.1671 .

minor isomer: Colorless oil (11 mg, 17.3\%), $\mathrm{R}_{\mathrm{f}} 0.58$ (petroleum ether/ethyl acetate 10:1). ${ }^{1} \mathrm{H} \mathrm{NMR}(600 \mathrm{MHz}$, $\left.\mathrm{CDCl}_{3}\right) \delta$ 7.72-7.68 (m, $\left.2 \mathrm{H}\right), 7.44(\mathrm{~m}, 2 \mathrm{H}), 7.40-7.37(\mathrm{~m}, 3 \mathrm{H}), 7.35(\mathrm{~m}, 2 \mathrm{H}), 7.28(\mathrm{~m}, 1 \mathrm{H}), 5.36(\mathrm{~d}, J 1.6 \mathrm{~Hz}, 1 \mathrm{H})$, $5.24(\mathrm{~d}, J 1.1 \mathrm{~Hz}, 1 \mathrm{H}), 4.37$ (dd, J 6.9, $3.4 \mathrm{~Hz}, 1 \mathrm{H}), 3.18(\mathrm{dt}, J$ 10.3, $6.9 \mathrm{~Hz}, 1 \mathrm{H}), 2.92(\mathrm{dd}, J 14.1,7.5 \mathrm{~Hz}, 1 \mathrm{H})$, $2.72(\mathrm{dd}, J$ 14.1, $7.3 \mathrm{~Hz}, 1 \mathrm{H}), 1.94-1.80(\mathrm{~m}, 2 \mathrm{H}), 1.74-1.60(\mathrm{~m}, 2 \mathrm{H}), 1.41-1.32(\mathrm{~m}, 1 \mathrm{H}), 1.22-1.15(\mathrm{~m}, 2 \mathrm{H}) .{ }^{13} \mathrm{C}$ NMR $\left(150 \mathrm{MHz}, \mathrm{CDCl}_{3}\right) \delta 164.1,145.8,140.9,129.9,129.4,128.8,128.4,127.4,126.8,126.3,115.0,82.5,77.2$, 77.0, 76.8, 45.2, 38.9, 35.1, 26.5 (d, J $13.1 \mathrm{~Hz}), 22.9$. HRMS (ESI) : $\mathrm{m} / \mathrm{z}$ calcd for $\mathrm{C}_{22} \mathrm{H}_{23} \mathrm{NONa}^{+}(\mathrm{M}+\mathrm{Na})^{+}$ 340.1672 , found 340.1673 .

Methyl-2-methylene-4-(3-phenyl-4,5-dihydroisoxazol-5-yl)butanoate (4a). Yellow oil (29 $\mathrm{mg}, 51 \%), R_{\mathrm{f}} 0.22$ (petroleum ether/ethyl acetate 10:1). ${ }^{1} \mathrm{H}$ NMR (500 MHz, $\left.\mathrm{CDCl}_{3}\right) \delta 7.69-7.62(\mathrm{~m}, 2 \mathrm{H}), 7.42-7.37(\mathrm{~m}, 3 \mathrm{H}), 6.20$ (s, $1 \mathrm{H}), 5.63(\mathrm{~d}, J 1.0 \mathrm{~Hz}, 1 \mathrm{H}), 4.82-4.68(\mathrm{~m}, 1 \mathrm{H}), 3.76(\mathrm{~s}, 3 \mathrm{H}), 3.42(\mathrm{dd}, J$ 16.5, $10.4 \mathrm{~Hz}, 1 \mathrm{H}), 3.01$ (dd, J 16.5, $7.9 \mathrm{~Hz}, 1 \mathrm{H}), 2.57-2.49(\mathrm{~m}, 1 \mathrm{H}), 2.49-2.41(\mathrm{~m}, 1 \mathrm{H}), 1.98-1.89(\mathrm{~m}, 1 \mathrm{H}), 1.84(\mathrm{~m}, 1 \mathrm{H}) .{ }^{13} \mathrm{C} \mathrm{NMR}\left(125 \mathrm{MHz} \mathrm{CDCl}_{3}\right)$ $\delta$ 167.4, 156.4, 139.4, 132.2, 130.0, 129.7, 129.2, 128.7, 128.4, 128.3, 127.0, 126.6, 126.3, 125.7, 117.0, 80.5, 77.3, 77.0, 76.8, 51.9, 39.9, 34.2, 28.1. HRMS (ESI): $\mathrm{m} / \mathrm{z}$ calcd for $\mathrm{C}_{15} \mathrm{H}_{17} \mathrm{NO}_{3} \mathrm{Na}^{+}(\mathrm{M}+\mathrm{Na})^{+} 282.1106$, found 282.1109.

Ethyl-2-methylene-4-(3-phenyl-4,5-dihydroisoxazol-5-yl)butanoate (4b). Colorless oil (34 mg, 62\%), $R_{f} 0.26$ (petroleum ether/ethyl acetate 10:1). ${ }^{1} \mathrm{H}$ NMR (500 MHz, $\left.\mathrm{CDCl}_{3}\right) \delta$ 7.68-7.64 (m, $\left.2 \mathrm{H}\right), 7.42-7.37(\mathrm{~m}, 3 \mathrm{H}), 6.19$ (d, J 0.4 Hz, 1 H), 5.61 (d, J 1.2 Hz, 1 H), 4.81-4.71 (m, 1 H), 4.21 (q, J 7.1 Hz, 2 H), $3.42(d d, J 16.5,10.4$ Hz, 1 H), 
$3.01(d d, J$ 16.5, $7.9 \mathrm{~Hz}, 1 \mathrm{H}), 2.56-2.48(\mathrm{~m}, 1 \mathrm{H}), 2.48-2.40(\mathrm{~m}, 1 \mathrm{H}), 1.94(\mathrm{~m}, 1 \mathrm{H}), 1.84(\mathrm{~m}, 1 \mathrm{H}), 1.30(\mathrm{t}, J 7.1$ $\mathrm{Hz}, 3 \mathrm{H}) .{ }^{13} \mathrm{C}$ NMR $\left(125 \mathrm{MHz}, \mathrm{CDCl}_{3}\right) \delta 167.0,156.4,139.7,130.0,129.7,129.1,128.7,128.4,126.6,126.3$, 125.4, 80.6, 77.3, 77.0, 76.8, 60.7, 39.9, 34.2, 28.1, 14.2. HRMS (ESI): $m / z$ calcd for $\mathrm{C}_{16} \mathrm{H}_{19} \mathrm{NO}_{3} \mathrm{Na}^{+}(\mathrm{M}+\mathrm{Na})^{+}$ 296.1263 , found 296.1265 .

tert-Butyl-2-methylene-4-(3-phenyl-4,5-dihydroisoxazol-5-yl)butanoate (4c). Yellow oil (31 mg, $51 \%), R_{f} 0.36$ (petroleum ether/ethyl acetate 10:1). ${ }^{1} \mathrm{H}$ NMR $\left(500 \mathrm{MHz}, \mathrm{CDCl}_{3}\right) \delta$ 7.69-7.64 (m, $\left.2 \mathrm{H}\right), 7.44-7.34(\mathrm{~m}, 3 \mathrm{H}), 6.09$ (d, J $1.3 \mathrm{~Hz}, 1 \mathrm{H}), 5.54$ (d, J $1.3 \mathrm{~Hz}, 1 \mathrm{H}), 4.76$ (dtd, J 10.4, 7.4, 5.9 Hz, $1 \mathrm{H}$ ), 3.42 (dd, J 16.5, $10.4 \mathrm{~Hz}, 1 \mathrm{H}$ ), 3.01 (dd, J 16.5, 7.9 Hz, $1 \mathrm{H}), 2.53-2.34(\mathrm{~m}, 2 \mathrm{H}), 1.94(\mathrm{~m}, 1 \mathrm{H}), 1.83(\mathrm{~m}, 1 \mathrm{H}), 1.49(\mathrm{~s}, 9 \mathrm{H}) .{ }^{13} \mathrm{C} \mathrm{NMR}\left(125 \mathrm{MHz}, \mathrm{CDCl}_{3}\right)$ $\delta 156.4,141.2,130.0,129.8,128.7,126.6,124.5,80.7$ (d, J $5.7 \mathrm{~Hz}), 77.3,77.0,76.8,39.9,34.3,28.1$. HRMS (ESI): $m / z$ calcd for $\mathrm{C}_{18} \mathrm{H}_{23} \mathrm{NO}_{3} \mathrm{Na}^{+}(\mathrm{M}+\mathrm{Na})^{+} 324.1576$, found 324.1572.

2-Methylene-4-(3-phenyl-4,5-dihydroisoxazol-5-yl)butanenitrile (4d). Orange solid (24 mg, 52\%), mp 44-45 ${ }^{\circ} \mathrm{C}, \mathrm{R}_{\mathrm{f}} 0.14$ (petroleum ether/ethyl acetate 10:1). ${ }^{1} \mathrm{H}$ NMR $\left(500 \mathrm{MHz}, \mathrm{CDCl}_{3}\right) \delta$ 7.72-7.61 (m, $\left.2 \mathrm{H}\right), 7.46-7.36(\mathrm{~m}$, $3 \mathrm{H}), 5.90(\mathrm{~s}, 1 \mathrm{H}), 5.82(\mathrm{~s}, 1 \mathrm{H}), 4.76(\mathrm{dtd}, J$ 10.5, 7.7, $4.9 \mathrm{~Hz}, 1 \mathrm{H}), 3.48(\mathrm{dd}, J$ 16.5, $10.4 \mathrm{~Hz}, 1 \mathrm{H}), 3.02$ (dd, J 16.5, $7.5 \mathrm{~Hz}, 1 \mathrm{H}), 2.57-2.39(\mathrm{~m}, 2 \mathrm{H}), 2.03-1.86(\mathrm{~m}, 2 \mathrm{H}) .{ }^{13} \mathrm{C} \mathrm{NMR}\left(125 \mathrm{MHz}, \mathrm{CDCl}_{3}\right) \delta 156.5,131.3,130.2$, $129.4,128.8,126.7,122.0,118.4,79.4,77.3,77.0,76.8,40.2,33.4,30.9$. HRMS (ESI): $\mathrm{m} / \mathrm{z}$ calcd for $\mathrm{C}_{14} \mathrm{H}_{14} \mathrm{~N}_{2} \mathrm{ONa}^{+}(\mathrm{M}+\mathrm{Na})^{+} 249.0998$, found 249.1000 .

2-Methylene-1-phenyl-4-(3-phenyl-4,5-dihydroisoxazol-5-yl)butan-1-one (4e) White solid (20 mg, 32\%) ; mp 87-88 ${ }^{\circ} \mathrm{C}, \mathrm{R}_{\mathrm{f}} 0.21$ (petroleum ether/ethyl acetate 10:1). ${ }^{1} \mathrm{H}$ NMR $\left(500 \mathrm{MHz}, \mathrm{CDCl}_{3}\right) \delta 7.75(\mathrm{~m}, 2 \mathrm{H}), 7.70-7.64(\mathrm{~m}$, $2 \mathrm{H}), 7.57-7.52(\mathrm{~m}, 1 \mathrm{H}), 7.47-7.37(\mathrm{~m}, 5 \mathrm{H}), 5.95(\mathrm{~s}, 1 \mathrm{H}), 5.68(\mathrm{~s}, 1 \mathrm{H}), 4.86-4.76(\mathrm{~m}, 1 \mathrm{H}), 3.45$ (dd, J 16.5, 10.4 $\mathrm{Hz}, 1 \mathrm{H}), 3.05$ (dd, J 16.5, $7.9 \mathrm{~Hz}, 1 \mathrm{H}), 2.72-2.58(\mathrm{~m}, 2 \mathrm{H}), 2.03-1.85(\mathrm{~m}, 2 \mathrm{H}) .{ }^{13} \mathrm{C} \mathrm{NMR}\left(125 \mathrm{MHz}, \mathrm{CDCl}_{3}\right) \delta 198.1$, 156.5, 147.0, 137.7, 132.3, 130.0, 129.8, 129.5, 128.7, 128.2, 126.7, 80.7, 77.3, 77.0, 76.8, 40.0, 34.0, 28.5. HRMS (ESI): $m / z$ calcd for $\mathrm{C}_{20} \mathrm{H}_{19} \mathrm{NO}_{2} \mathrm{Na}^{+}(\mathrm{M}+\mathrm{Na})^{+} 328.1308$, found 328.1307.

Phenyl-3-(3-phenyl-4,5-dihydroisoxazol-5-yl)propan-1-one (5a). White solid (28 mg, 50\%); $\mathrm{mp} 78-79{ }^{\circ} \mathrm{C}, \mathrm{R}_{\mathrm{f}}$ 0.12 (petroleum ether/ethyl acetate 10:1). ${ }^{1} \mathrm{H}$ NMR $\left(500 \mathrm{MHz}, \mathrm{CDCl}_{3}\right) \delta 8.04-7.92(\mathrm{~m}, 2 \mathrm{H}), 7.70-7.62(\mathrm{~m}, 2 \mathrm{H})$, 7.61-7.51 (m, $1 \mathrm{H}), 7.46$ (dd, J 10.6, $4.7 \mathrm{~Hz}, 2 \mathrm{H}), 7.43-7.38(\mathrm{~m}, 3 \mathrm{H}), 4.86(\mathrm{~m}, 1 \mathrm{H}), 3.48(\mathrm{dd}, J 16.5,10.3 \mathrm{~Hz}, 1$ $\mathrm{H}), 3.23(\mathrm{t}, J 7.2 \mathrm{~Hz}, 2 \mathrm{H}), 3.09-3.01(\mathrm{~m}, 1 \mathrm{H}), 2.19(\mathrm{~m}, 1 \mathrm{H}), 2.14-2.02(\mathrm{~m}, 1 \mathrm{H}) .{ }^{13} \mathrm{C} \mathrm{NMR}\left(125 \mathrm{MHz}, \mathrm{CDCl}_{3}\right) \delta$ $199.3,156.7,136.8,133.2,130.1,129.6,128.7,128.1,126.6,80.2,77.3,77.0,76.8,40.3,34.4$, 29.7. HRMS (ESI): $m / z$ calcd for $\mathrm{C}_{18} \mathrm{H}_{17} \mathrm{NO}_{2} \mathrm{Na}^{+}(\mathrm{M}+\mathrm{Na})^{+} 302.1157$, found 302.1138 .

\section{Acknowledgements}

This work was supported by Natural Science Foundation of Zhejiang province (LY21B020008) and National Key Research and Development Program of China (2017YFC0210900).

\section{Supplementary Material}

Supplemental material such as substrate preparation, optimization details, references and ${ }^{1} \mathrm{H} N M R$, ${ }^{13} \mathrm{C} \mathrm{NMR},{ }^{19} \mathrm{~F}$ NMR spectra for compounds 3a-3p, 4a-4e and $5 a$ for this article is available online.

\section{References}

1. Lan, X.-W.; Wang, N.-X.; Xing, Y. Eur. J. Org. Chem. 2017, 5821-5851. https://doi.org/10.1002/ejoc.201700678

2. Li, Y.; Wu, D.; Cheng, H. G.; Yin, G. Angew. Chem. Int. Ed. 2020, 59, 7990-8003. 
https://doi.org/10.1002/anie.201913382

3. Jensen, K. H.; Sigman, M. S. Org. Biomol. Chem. 2008, 6, 4083-4088.

https://doi.org/10.1039/b813246a

4. McDonald, R. I.; Liu, G.; Stahl, S. S. Chem. Rev. 2011, 111, 2981-3019.

https://doi.org/10.1021/cr100371y

5. Egami, H.; Sodeoka, M. Angew. Chem. Int. Ed. 2014, 53, 8294-8308.

https://doi.org/10.1002/anie.201309260

6. Egami, H.; Sodeoka, M. Angew. Chem. Int. Ed. 2014, 126, 8434-8449.

https://doi.org/10.1002/ange.201309260

7. Merino, E.; Nevado, C. Chem. Soc. Rev. 2014, 43, 6598-6608.

https://doi.org/10.1039/c4cs00025k

8. Coombs, J. R.; Morken, J. P. Angew. Chem. Int. Ed. 2016, 55, 2636-2649.

https://doi.org/10.1002/anie.201507151

9. John, R.; Coombs, J.; Morken, P. Angew. Chem. Int. Ed. 2016, 128, 2682-2696.

https://doi.org/10.1002/ange.201507151

10. Yin, G.; Mu, X.; Liu, G. Acc Chem. Res. 2016, 49, 2413-2423.

https://doi.org/10.1021/acs.accounts.6b00328

11. Derosa, J.; van der Puyl, V. A.; Tran, V. T.; Liu, M.; Engle, K. M. Chem. Sci. 2018, 9, 5278-5283. https://doi.org/10.1039/c8sc01735b

12. Giri, R.; Kc, S. J. Org. Chem. 2018, 83, 3013-3022.

https://doi.org/10.1021/acs.joc.7b03128

13. Zhang, J. S.; Liu, L.; Chen, T.; Han, L. B. Chem. Asian J. 2018, 13, 2277-2291.

https://doi.org/10.1002/asia.201800647

14. Wang, Z. X.; Bai, X. Y.; Li, B. J. Chin. J. Chem. 2019, 37,1174-1180.

https://doi.org/10.1002/cjoc.201900308

15. Gu, Q.-S.; Li, Z.-L.; Liu, X.-Y. Acc. Chem. Res. 2020, 53, 170-181.

https://doi.org/10.1021/acs.accounts.9b00381

16. Li, Z.-L.; Fang, G.-C.; Gu, Q.-S.; Liu, X.-Y. Chem. Soc. Rev. 2020, 49, 32-48.

https://doi.org/10.1039/c9cs00681h

17. Melhado, A. D.; Brenzovich, W. E.; Lackner, A. D.; Toste, F. D. J. Am. Chem. Soc. 2010, 132, 8885-8887. https://doi.org/10.1021/ja1034123

18. Xie, Y.; Hu, J.; Xie, P.; Qian, B.; Huang, H. J. Am. Chem. Soc. 2013, 135, 18327-18330. https://doi.org/10.1021/ja410611b

19. Zhu, R.; Buchwald, S. L. J. Am. Chem. Soc. 2015, 137,8069-8077.

https://doi.org/10.1021/jacs.5b04821

20. Li, X.; Chen, K.; Tang, Y.; Zhu, H.; Chen, F.; Yan, X.; Xu, X. Synlett 2018, 29, 1634-1638. https://doi.org/10.1055/s-0037-1609968

21. Li, Y.; Lu, R.; Sun, S.; Liu, L. Org. Lett. 2018, 20, 6836-6839.

https://doi.org/10.1021/acs.orglett.8b02954

22. Gao, M.; Gan, Y.; Xu, B. Org. Lett. 2019, 21, 7435-7439.

https://doi.org/10.1021/acs.orglett.9b02748

23. Li, X.; Wang, X.; Wang, Z.; Yan, X.; Xu, X. ACS Sustain. Chem. Eng. 2019, 7, 1875-1878.

https://doi.org/10.1021/acssuschemeng.8b06096

24. Ura, Y. Synthesis 2021, 53 (05), 848-860. 


\section{https://doi.org/10.1055/s-0040-1706570}

25. Li, X.-T.; Gu, Q.-S.; Dong, X.-Y.; Meng, X.; Liu, X.-Y. Angew. Chem. Int. Ed. 2018, 57, 7668-7672. https://doi.org/10.1002/anie.201804315

26. Li, X.-T.; Lv, L.; Wang, T.; Gu, Q.-S.; Xu, G.-X.; Li, Z.-L.; Ye, L.; Zhang, X.-H.; Cheng, G.-J.; Liu, X.-Y. Chem. 2020, 6, 1692-1706.

https://doi.org/10.1016/j.chempr.2020.03.024

27. Hewitt, J. F. M.; Williams, L.; Aggarwal, P.; Smith, C. D.; France D. J. Chem. Sci. 2013, 4, 3538-3543. https://doi.org/10.1039/c3sc51222c

28. Quinn, R. K.; Schmidt, V. A.; Alexanian, E. J. Chem. Sci. 2013, 4, 4030-4034. https://doi.org/10.1039/c3sc51466h

29. Han, W. J.; Wang, Y. R.; Zhang, J. W.; Chen, F.; Zhou, B.; Han, B. Org. Lett. 2018, 20, 2960-3963. https://doi.org/10.1021/acs.orglett.8b01004

30. Liu, R.-H.; Wei, D.; Han, B.; Yu, W. ACS Catal. 2016, 6, 6525-6530. https://doi.org/10.1021/acscatal.6b02065

31. Zhu, L.; Wang, G.; Guo, Q.; Xu, Z.; Zhang, D.; Wang, R. Org. Lett. 2014, 16, 5390-5393. https://doi.org/10.1021/ol502624z

32. Li, X.; Ding, Y.; Qian, L.; Gao, Y.; Wang, X.; Yan, X.; Xu, X. J. Org. Chem. 2019, 84, 12656-12663. https://doi.org/10.1021/acs.joc.9b02031

33. Kaur, K.; Kumar, V.; Sharma, A. K.; Gupta, G. K. Eur. J. Med. Chem. 2014, 77, 121-133. https://doi.org/10.1016/j.ejmech.2014.02.063

34. Castellano, S.; Kuck, D.; Viviano, M.; Yoo, J.; López-Vallejo, F.; Conti, P.; Tamborini, L.; Pinto, A.; MedinaFranco, J. L.; Sbardella, G. J. Med. Chem. 2011, 54, 7663-7677.

https://doi.org/10.1021/jm2010404

35. Jewett, J. C.; Sletten, E. M.; Bertozzi, C. R. J. Am. Chem. Soc. 2010, 132, 3688-3690. https://doi.org/10.1021/ja100014q

36. Jullien, N.; Makritis, A.; Georgiadis, D.; Beau, F.; Yiotakis, A.; Dive, V. J. Med. Chem. 2010, 53, 208-220. https://doi.org/10.1021/jm9010803

37. Poutiainen, P. K.; Palvimo, J. J.; Hinkkanen, A. E.; Valkonen, A.; Väisänen, T. K.; Laatikainen, R.; Pulkkinen, J. T. J. Med. Chem. 2013, 56, 1064-1073.

https://doi.org/10.1021/jm301516q

38. García-Reynaga, P.; Zhao, C.; Sarpong, R.; Casida, J. E. Chem. Res. Toxicol. 2013, 26, 514-516. https://doi.org/10.1021/tx400055p

39. Hwang, I. T.; Kim, H. R.; Jeon, D. J.; Hong, K. S.; Song, J. H.; Cho, K. Y. J. Agric. Food Chem. 2005, 53, 8639-8643.

https://doi.org/10.1021/jf051284f

40. Hwang, K.-H.; Lim, J.-S.; Kim, S.-H.; Jeon, M.-S.; Lee, D.-G.; Chung, K.-H.; Koo, S.-J.; Kim, J.-H.; J. Agric. Food Chem. 2013, 61, 9285-9292.

https://doi.org/10.1021/jf4025823

41. Kozikowski, A. P.; Stein, P. D. J. Am. Chem. Soc. 1982, 104, 4023-4026. https://doi.org/10.1021/ja00378a049

42. Arai, M. A.; Arai, T.; Sasai, H. Org. Lett. 1999, 1, 1795-1797. https://doi.org/10.1021/ol9902881

43. Arai, M. A.; Kuraishi, M.; Arai, T.; Sasai, H. J. Am. Chem. Soc. 2001, 123, 2907-2908. https://doi.org/10.1021/ja005920w 
44. Spannring, P.; Bruijnincx, P. C. A.; Weckhuysen, B. M.; Gebbink, R. J. M. K. RSC Adv. 2013, 3, 6606-6613. https://doi.org/10.1039/c3ra40324f

45. Liu, H.; Ge, L.; Wang, D. X.; Chen, N.; Feng, C. Angew. Chem. Int. Ed. 2019, 58, 3918-3922. https://doi.org/10.1002/anie.201814308

46. Zhang, J.; Li, Y.; Xu, R.; Chen, Y. Angew. Chem. Int. Ed. 2017, 56, 12619-12623. https://doi.org/10.1002/anie.201707171

This paper is an open access article distributed under the terms of the Creative Commons Attribution (CC BY) license (http://creativecommons.org/licenses/by/4.0/) 